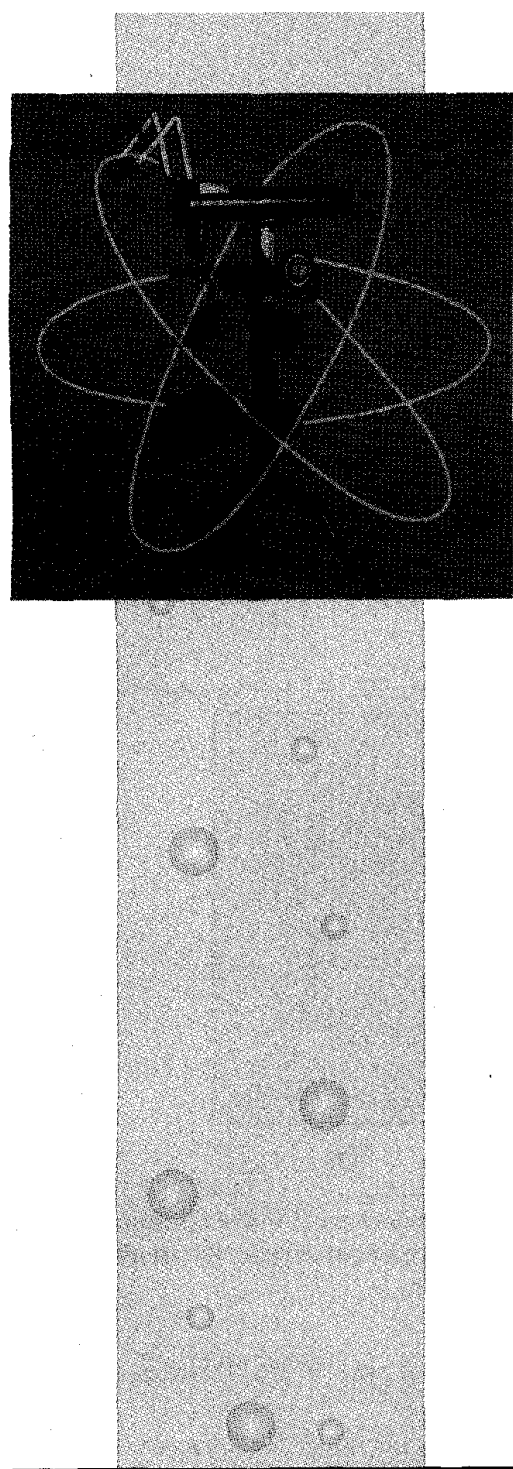

$\operatorname{lan}$ T. Young

Foculty of Applied Physics

Delft University of Technology

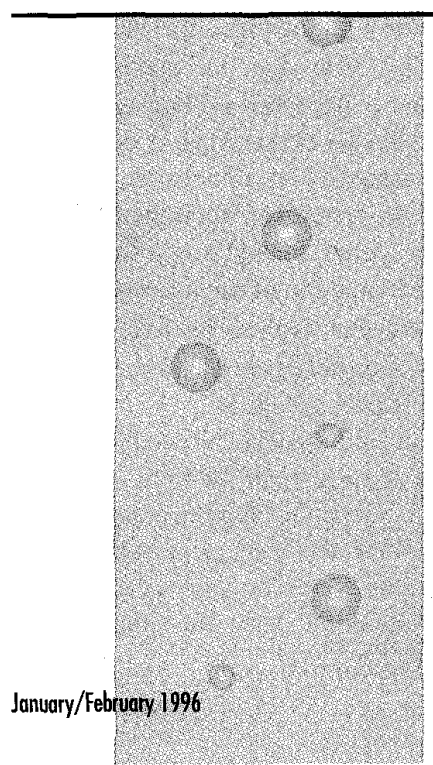

Quantitative Microscopy

W

hile light microscopy is almost 400 years old [1, 2], developments of the past decade have offered a variety of new mechanisms for examination of biological and material samples. These developments include exploitation of techniques such as confocal microscopy, scanning near field microscopy, standing wave microscopy, fluorescence lifetime microscopy, and two-photon microscopy. (See, for example, recent issues of Bioimaging and Journal of Microscopy.) In biology, advances in molecular biology and biochemistry have made it possible to selectively tag (and thus make visible) specific parts of cells, such as actin molecules, or sequences of DNA of 1000 base pairs or longer. In sensor technology, modern charge-coupled devices (CCD) cameras are capable of achieving high spatial resolution and high sensitivity measurements of signals in the optical microscope. In computer processing, we have learned how to process digitized images so as to extract meaningful measurements of "analog" quantities given digital data.

The applications that motivate and exploit these developments can be divided into those in which the goal is the production of images that are to be used as images by human observers and those where the images are to be analyzed to produce data for human interpretation. In the former, where we can speak of "specimen in" $\rightarrow$ "image out," the issue is image processing; in the latter, where we can speak of "specimen in" $\rightarrow$ "data out," the issue is image analysis. As we hope to demonstrate in this paper, the two different applications, processing and analysis, can lead to different sets of conclusions in the choice of algorithms and technical constraints such as sampling frequency.

In the case of image analysis, we can also make a clear distinction between problems of detection and problems of estimation. An example of a detection problem might be finding the spots produced in a cell nucleus by molecular probes that are specific for the DNA on either chromosome number 1 or chromosome number 7. Using fluorescent dyes to color chromosome 1 green and chromosome 7 orange-red, and to color the entire DNA content blue (see Fig. 1a), the central problem becomes the detection of the colored dots followed by simple counting.
As a second example, we consider the measurement of the amount of DNA in each cell, in order to build a profile of the DNA distribution in a population of cells. In Fig. 1b we see cell nuclei that have been stained with a quantitative (stoichiometric) staining reagent (Feulgen) and where the amount of stain per pixel is proportional to the DNA content per pixel.

\section{Waves and Photons}

Modern physics has taught us that there are two inter-related ways of describing light -as waves and as a collection of massless particles, photons. Both of these descriptions are necessary in order to understand the properties and limitations of quantitative microscopy.

Waves - The wave description leads naturally to a consideration of the wavelength of light being used, $\lambda$, and the diffraction limits of modern microscope lenses. A modern well-designed, aberration "free" microscope lens may be characterized as an linear shift-invariant system with a point spread function (PSF), followed by a pure magnification system as shown in Fig. 2 [3].

The form of the PSF is circularly symmetric and given by:

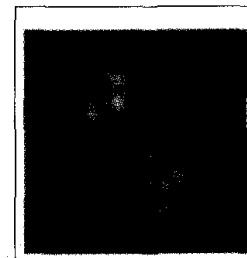

(a)

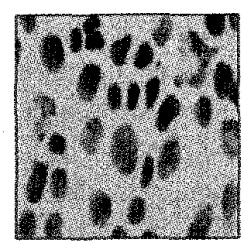

(b)
1. (a) Human lymphocytes stained with fluorescent dyes DAPI (blue), Spectrum Green, and Spectrum Orange, to reveal total DNA content, the centromeric DNA of chromosome 1 , and the centromeric DNA of chromosome 7, respectively. Spectrum Green and Spectrum Orange are registered trademarks of Vysis Corp., Downers Grove, IL. (b) Human tissue sampled with the absorptive dye Feulgen, which is quantitative for DNA content. The optical density pixel is proportional to the DNA content per pixel. 


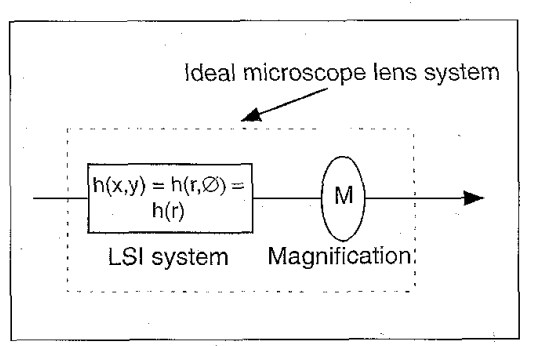

2. An ideal microscope lens has a point spread function that is circularly symmetric $h(r)$. This is followd by a magnification factor, $M$. Typical values of $M$ are 25,40 and 63 times. Note that the total lens system is not shift invariant because moving the input image (in space) by a distance $\Delta x$ will cause the output image to move by $M \Delta x$ instead of $\Delta x$.

$$
h(r)=\left(2 \frac{J_{1}(a r)}{r}\right)^{2} \text { with } a=\frac{2 \pi \cdot N A}{\lambda}
$$

where $J_{1}(\cdot)$ is a Bessel function of the first kind. We see that for this ideal case only two parameters are of consequence - the wavelength of light, $\lambda$, and the numerical aperture of the lens, NA. The NA of the lens measures its ability to collect light, and is given by $n \cdot \sin (\theta)$, with $n$ the index of refraction of the medium between the lens and the specimen, and $\theta$ the angle of acceptance of the microscope lens. Typical values of $n$ are 1.0 (air), 1.3 (water), and 1.5 (immersion oil). A maximum value for $\theta$ is about $69^{\circ}$, which leads to values of $N A$ that are less than 1.0 in air and less than 1.4 in oil.

The optical transfer function (OTF) of an ideal circularly-symmetric microscope lens can be calculated. Because of the circular symmetry, $H\left(\omega_{\mathrm{x}}, \omega_{\mathrm{y}}\right)=F\{h(x, y)\}$ $=F\{h(r)\}=H\left(\omega_{x}\right)$, where $F\{\bullet\}$ is the Fourier transform operation. The OTF is given by:

$$
\begin{aligned}
& H\left(\omega_{r}\right)= \\
& \left\{\begin{array}{c}
\frac{2}{\pi a^{2}}\left[\cos ^{-1}\left(\frac{\omega_{r}}{2 a}\right)-\left(\frac{\omega_{r}}{2 a}\right) \sqrt{1-\left(\frac{\omega_{r}}{2 a}\right)^{2}}\right]_{\omega_{r}>2 a} \leq 2 a \\
0
\end{array}\right.
\end{aligned}
$$

The PSF, $h(r)$, and the OTF, $H((r)$, are shown in Fig. 3. It is the PSF that gives rise to the well-known Airy disk [4].

An important feature of the OTF is that it is bandlimited. That is, there exists a frequency, $\omega_{\mathfrak{c}}$, such that $\left|H\left(\omega_{\mathrm{c}}\right)\right| \equiv 0$ for $|\omega|$ $>\omega_{c}$. This cutoff frequency is given by:

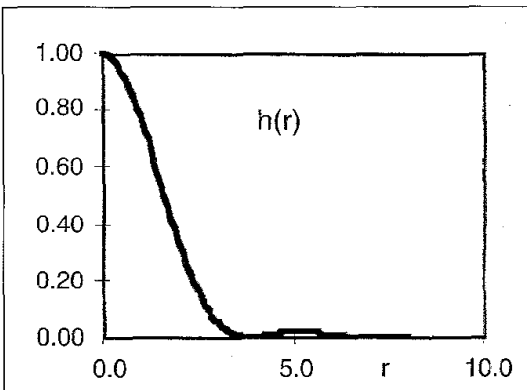

(a)

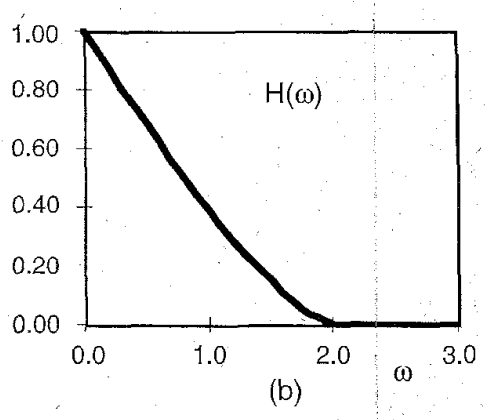

3. (a) Point spread function (PSF) and h(r) of an ideal microscope lens. Optical Transfer Function (OTF), H( $\left.\omega_{r}\right)$ of the ideal microscope lens. Both are evaluated for $\mathrm{a}=1$ in Eqs. 1 and 2.

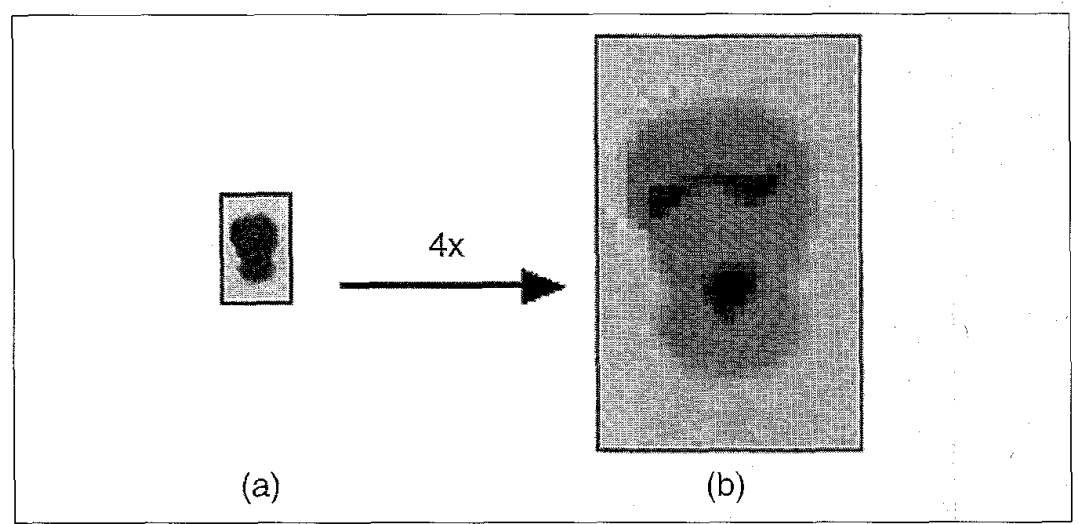

4. (a) Small metaphase chromosome stained with the absorptive dye Giemsa in order to reveal the band structure. (b) A 4 times enlargement reveals the small numer of pixels involved in the digital image representation.

$$
\omega_{c}=2 a=\frac{4 \pi \cdot N A}{\lambda}
$$

For green light with a wavelength of $500 \mathrm{~nm}$, and using an oil-immersion lens with $N A=1.4$, this corresponds to a cutoff frequency of 5.6 cycles per $\mu \mathrm{m}$ (microns). The Nyquist sampling theorem would then imply that an image to be analyzed after sampling would require a minimum sampling frequency of $2 \omega_{c}$. In this example this implies 11.2 samples per micron or a maximum distance of 0.089 microns between samples. While this very fine sampling might appear to be "overkill", we should remember that biological samples do contain arbitrarily small physical details, and thus the Fourier spectrum is not limited by the input physical signal (the specimen) but rather by the diffraction limits of the microscope objective (a low-pass filter). As a practical consequence, the examination of a small human metaphase chromosome, which is only about 1 by 2 microns leads to a digital image of only 15 by 30 pixels, as shown in Fig. 4

Another direct consequence of the bandlimited nature of the microscope optics can be found in procedures for autofocusing as well as for understanding the issue of depth-of-focus. When an image specimen with Fourier spectrum $\mathrm{I}\left(\omega_{\mathrm{x}}, \omega_{\mathrm{y}}\right)$ is passed through a microscope with an OTF given by $H\left(\omega_{\mathrm{x}}, \omega_{\mathrm{y}}\right)$, an output image $\mathrm{O}\left(\omega_{\mathrm{x}}, \omega_{\mathrm{y}}\right)=I\left(\omega \mathrm{x}, \omega_{\mathrm{y}}\right) \bullet H\left(\omega_{\mathrm{x}}, \omega_{\mathrm{y}}\right)$ is produced. The act of focusing or defocusing the microscope does not change the spectrum of the specimen, but rather the OTF. In other words, the OTF is a function of the $z$-axis position of the microscope. We can make this explicit by writing $H\left(\omega_{\mathrm{x}}\right.$, $\left.\omega_{y}, \Delta z\right)$. A typical example of this dependency is shown in Fig. 5.

It is clear that independent of the focus, $H(\omega=0, \Delta z)=1.0$; all the light that enters the microscope through the objective lens is assumed to leave the microscope through the ocular or camera lens. At the bandlimit of the lens, the amplitude of 


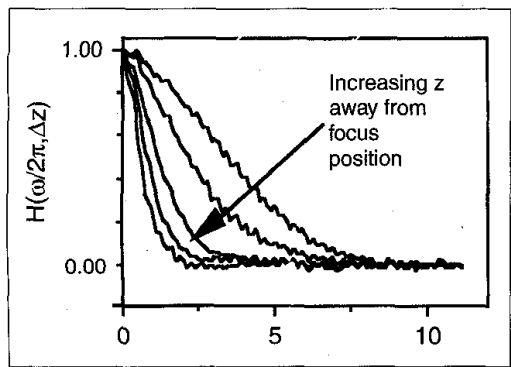

5. As we move away from optimum focus, as $\Delta z$ increases, the $H\left(\omega_{r} / 2 \pi\right)$ sags. These measured data describe a 60 times lens with an NA of 1.4 (oil immersion), and a wavelength of $400 \mathrm{~nm}$ (blue). The cutoff frequency, $\mathbf{f}_{\mathrm{c}}$ (from Eq. 3) should be 7.0 cycles per $\mu \mathrm{m}$

$H\left(\omega=\omega_{c}, \Delta z\right) \approx 0.0$, again independent of focus. Thus, autofocus algorithms can only expect to work well when they examine midband frequencies $\left(\omega_{\mathrm{r}} / 2 \mathrm{a} \approx 0.75\right.$, as seen in Fig. 3b) and when the input signal spectrum, $I\left(\omega_{\mathrm{x}}, \omega_{\mathrm{y}}\right)$, contains a sufficient amount of energy in that spectral found in [5].

Further, the depth-of-focus - that distance $\Delta z$ over which the image specimen can be expected to be observed without significant optical aberration -can be derived from considerations of wave optics and shown to be $[6,7]$ :

$$
\Delta z=\frac{\lambda}{4 \cdot n\left(1-\sqrt{1-\left(\frac{N A}{n}\right)^{2}}\right)}
$$

Again using the typical values of $\lambda=$ $500 \mathrm{~nm}, N A=1.4$, and $n=1.5$, we arrive at a depth-of-focus of $\Delta z=0.13 \mu \mathrm{m}$, a very thin region of critical focus.

Photons-A second and equally important aspect of the physical signal that we observe is the quantum nature of light. Assuming for the moment an ideal situation, a single photon that arrives at a single CCD camera pixel may have been transmitted through a specimen (as in absorptive microscopy) or may have been emitted by a fluorescent dye molecule. In either case, that single photon for $\lambda=500$ $\mathrm{nm}$ will carry an energy of $E=\mathrm{h} v=\mathrm{hc} / \lambda$ infinitesimal amount of energy, modern CCD cameras are sensitive enough to be able to count individual photons. The real problem arises, however, from the fundamentally statistical nature of photon proband. A complete analysis of this can be $=3.97 \cdot 10^{-19} \mathrm{~J}$. While this is a seemingly

duction. We cannot assume that in a given pixel in two consecutive but independent observation intervals of length $T$, that the same number of photons will be counted. Photon production is governed by the laws of quantum physics, which restricts us to talking about an average number of photons within a given observation window. The probability distribution of photons in an observation window of length $T$ seconds is known to be Poisson [8]. That is, the probability of $p$ photons in an interval of length $T$ is given by:

$$
P(p \mid \gamma, T)=\frac{(\gamma T)^{p} e^{-\gamma T}}{p !}
$$

where $\gamma$ is the rate or intensity parameter measured in photons per second. It is critical to understand that, even if there were no other noise sources in the imaging chain, the statistical fluctuations associated with photon counting over a finite time interval, $T$, would still lead to a finite signal-to-noise ratio (SNR). If we express this SNR as $20 \log _{10}(\mu / \sigma)$, then, due to the fact that the average value of a Poisson process is given by $\mu=\gamma$, and the standard deviation is given by $\sigma=\sqrt{\gamma}$ we have that SNR $=10 \log _{10}(\gamma)$.

In this context, it is important to understand that the three traditional assumptions about the relationship between signal and noise do not hold:

- noise is not independent of the signal;

- noise is not Gaussian, and;

- noise is not additive.

Techniques that have been developed to deal with noisy images, under the traditional assumptions - techniques for enhancement, restoration, segmentation,

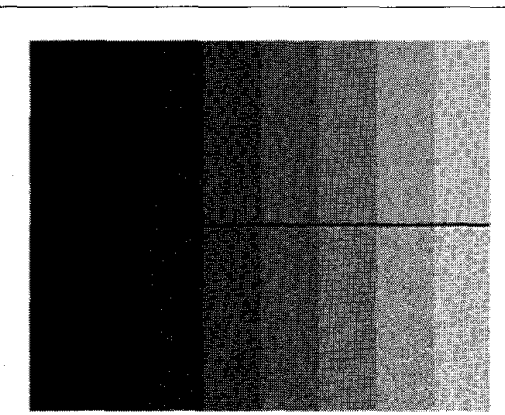

(a) and measurement-must be reexamined before they can be used with these types of images.

\section{Camera Evaluation}

Thinking in terms of photons has a direct effect on the evaluation of alternative camera systems for quantitative microscopy. When a photon strikes the photosensitive surface of a CCD, it may or may not cause a photoelectron to be collected in the potential well. The probability of this happening is associated with the quantum efficiency of the material (usually silicon) and the energy (wavelength) of the photon. Typical values for the quantum efficiency for silicon are around $50 \%$, increasing towards the infrared and decreasing towards the blue end of the spectrum. But each photoelectron that is produced comes from one photon, so that photoelectrons (as well as photons) have a Poisson distribution. If a CCD well has a finite capacity for photoelectrons, $C$, then the maximum possible signal will be $C$ and the standard deviation will be $\sqrt{C}$. This means that the maximum SNR per pixel will be limited to $\mathrm{SNR}_{\max }=10$ $\log _{10}(\mathrm{C})$. Thus, even if all other sources of noise are negligible compared to the fundamental fluctuations in the photon counts, the SNR will be limited by the CCD well capacity. If we choose to perform on-chip integration after the well is full, then we will only achieve blooming - the leaking of the overfull well into other nearby wells. For three well-known CCD chips, these limits are given in Table 1.

Each of these chips, when integrated into a well-designed camera, is capable of achieving these theoretical maximum

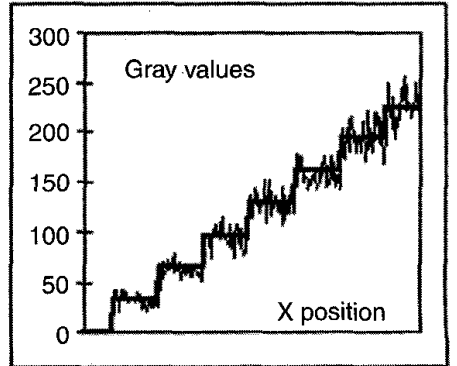

(b)
6. (a) Top: gray level step wedge with 8 levels. Bottom: the same step wedge as if each pixel were contaminated with Poisson noise and the rate parameter, $\gamma$, were the original pixel value (with $T=1$ in Eq. 5). (b) Heavy curve shows a horizontal line through the uncontaminated step wedge; thin line shows the result of the Poisson noise contamination. 


\begin{tabular}{|l|l|l|l|}
\hline \multicolumn{3}{|c|}{ Table 1: Characteristics Per Pixel of Some Well-known CCD Chips } \\
\hline Chip & $\begin{array}{l}\text { Pixel Size } \\
\mu \mathrm{m} \times \mu \mathrm{m}\end{array}$ & $\begin{array}{l}\text { Capacity } \\
\text { phoioelectrons }\end{array}$ & $\begin{array}{l}\text { SNRmax } \\
\mathrm{dB}\end{array}$ \\
\hline Kodak KAF 1400 & $6.8 \times 6.8$ & 32,000 & 45 \\
\hline Sony PA-93 & $11.0 \times 11.0$ & 80,000 & 49 \\
\hline Thompson TH 7882 & $23.0 \times 23.0$ & 400,000 & 56 \\
\hline
\end{tabular}

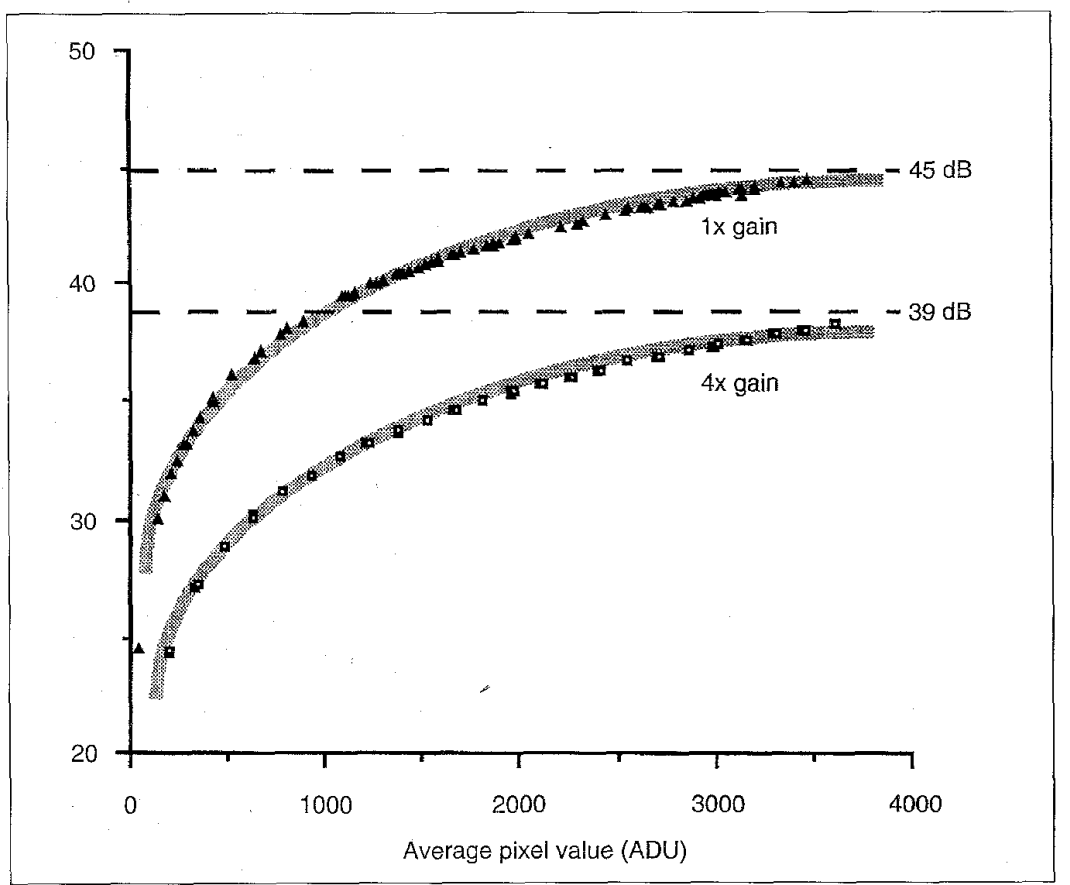

7. SNR as a function of the recorded brighness image for a cooled Photometrics KAF 1400 camera. The data were collected in both the 1 and 4 times gain modes. The data follo a log function (shown in thick gray line) to the maximum well capacity of the CCD photoelement.

SNR values [9]. The invariant among these three chips is the photoelectron capacity per square micron. If we think of the well as having a volume given by the cross-sectional area times the depth, then the capacity per unit cross-sectional area for all three chips is about 700 photoelectrons $/ \mu \mathrm{m}^{2}$.

The verification that the SNR is photon-limited can be achieved by looking at the form of $\operatorname{SNR}(C)$ versus $C$. When the form is $\log (C)$, and the asymptotic value is that given in Table 1, then we can be confident that we are dealing with a welldesigned camera that is, in fact, limited only by photon noise. An example of this type of result for a Photometrics CC200 camera (Photometrics Corp., Tucson, AZ), based on the Kodak KAF 1400 chip, is given in Fig. 7 .

Using the Poisson model, it is also possible to determine the sensitivity of each camera. There is clearly a scale factor $(G)$ between photoelectrons $\left(\mathrm{e}^{-}\right)$and ADU. (An ADU is the step size of the A/D converter. That is, the difference between gray levels $k$ and $k+1$ ) Thus, the output $y$ (in $A D U$ ) and the input $x$ (in photoelectrons) are related by $\mathrm{y}=G \cdot \mathrm{x}$. It holds for any random variable that $\mathrm{E}\{y\}=\mathrm{E}\{G \cdot x\}$ $=G \cdot \mathrm{E}\{x\}$ and $\mathrm{V}\{y\}=\mathrm{V}\{G \cdot x\}=G^{2} \cdot \mathrm{V}\{x\}$, where $\mathrm{E}\{\cdot\}$ and $\mathrm{V}\{\cdot\}$ are the expectation and variance operators, respectively. Using the additional constraint that $x$ has a Poisson distribution gives $\mathrm{E}\{y\}=G \cdot \mathrm{E}\{x\}$ $=G \cdot \gamma$ and $\mathrm{V}\{y\}=G^{2} \cdot \mathrm{V}\{x\}=G^{2} \cdot \gamma$. This means that an estimate of the scale factor is given by $\mathrm{V}\{y\} / \mathrm{E}\{y\}=G$, independent of $\gamma$. The sensitivity, $S$, is simply $1 / G$. The sensitivity for the three chips mentioned above, in specific camera configurations, is given in Table 2 .

The extraordinary sensitivity of modern CCD cameras is clear from these data. In the $1 \mathrm{x}$ gain mode of the Photometrics KAF 1400 camera, only 8 photoelectrons (approximately 16 photons) separate two gray levels in the digital representation of the image. For the considerably less expensive Sony camera, only about 500 photons separate two gray levels.

There are, of course, other possible sources of noise. Specifically:

- Readout noise--which can be reduced to manageable levels by slow readout rates and proper electronics. At very low signal levels, however, readout noise can become a significant component in the overall SNR.

- Amplifier noise-which is negligible with well-designed electronics.

- Quantization noise - which is inherent in the digitization process and yields an additive noise with $\mathrm{SNR}=$ $6 b+11 \mathrm{~dB}$, where $b$ is the number of bits. For $b \geq 8$ bits, this means a SNR $\geq 59 \mathrm{~dB}$. Quantization noise can, therefore, be ignored when compared to the SNRs listed in Table 1, as the total SNR of a complete system is dominated by the smallest SNR.

- Dark current-An additional stochastic source of photoelectrons is thermal energy. Electrons can be freed from the material itself through thermal vibration and then, trapped in the CCD well, be indistinguishable from "true" photoelectrons. By cooling the CCD chip to around $-40^{\circ} \mathrm{C}$, it is possible to reduce significantly the number of "thermal electrons" that give rise to dark current. Clearly, as the integration time, $T$, increases, the number of thermal electrons increases. The probability distribution of thermal electrons is also a Poisson process, where the rate parameter is an increasing function of temperature. It is straightforward to measure this dark current and typical results for the three cameras are presented in Table 2 [9]. There are alternative techniques (to cooling) for suppressing dark current. These usually involve estimating the average dark current for the given integration time and then subtracting this value from the CCD pixel values before the $A / D$ converter. While this technique does reduce the dark current, it also reduces the dynamic range of the camera.

All of this is of more than of academic interest when we consider the strength of 
Table 2: Characteristics Per Pixel of Some Well-Known Cameras*

\begin{tabular}{|l|c|c|c|}
\hline \multicolumn{1}{|c|}{ Camera/Chip } & $\begin{array}{c}\text { Sensitivity } \\
\text { e-/ADU }\end{array}$ & $\begin{array}{c}\text { Dark Current } \\
\text { ADU/s }\end{array}$ & $\begin{array}{c}\text { Temperature } \\
\text { C }\end{array}$ \\
\hline $\begin{array}{l}\text { Photometrics/KAF } \\
1400\end{array}$ & 7.9 & .002 & -42 \\
\hline $\begin{array}{l}\text { Sony XC- } \\
77 \text { RRCE/PA-93 }\end{array}$ & 256.4 & .043 & +22 \\
\hline $\begin{array}{l}\text { Photometrics/TH } \\
7882\end{array}$ & 90.9 & .420 & -37 \\
\hline
\end{tabular}

*Both of the Photometrics cameras are cooled using Peltier elements. Both were evaluated in the $1 \times$ gain setting. The Sony camera is being used in integration mode, with integration time on the order of 3 to 4 seconds. (See [9].)

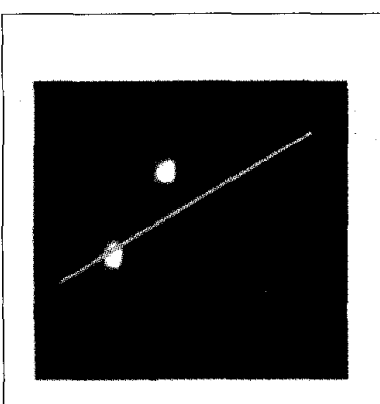

(a)

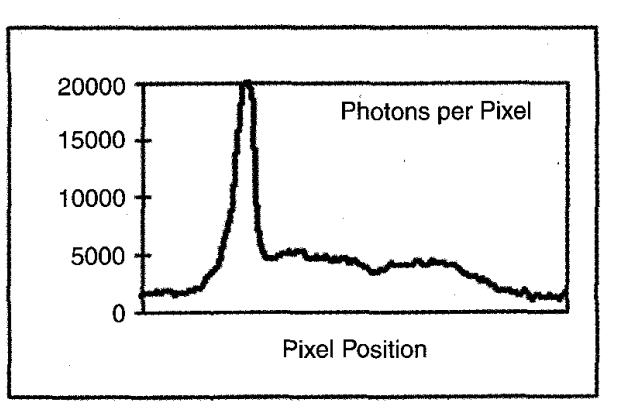

(b)

8. (a) Interphase nucleus stained for both general DNA (gray) and centromeric DNA (white dots). Exposure time was 4 s, with a Photmetrics KAF 1400 camera. (b) Number of photons per pixel along the yellow line in (a).

signals that are encountered in fluorescence microscopy. An example is shown in Fig. 8 [10].

\section{Sampling Density}

There are other sources of noise in a digital image besides noise contamination of the pixel brightness. The act of sampling-cutting up the image into rows and columns in 2D and rows, columns, and planes in 3D - is also an important source of noise, which is of particular significance when the goal is image analysis. The potential effect of this kind of noise can be illustrated with the relatively simple problem of measuring the area of a two dimensional object such as cell nucleus. It has been known for many years [11] that the best measure of the area of an "analog" object, given its digital representation, is simply to count the pixels associated with the object. The use of the term "best estimate" means that the estimate is unbiased (accurate) and that the variance goes to zero (precise) as the sampling density increases. We assume here that the pixels belonging to the object have been labeled, thus producing a binary representation of the object. The issue of using the actual gray values of the object pixels to estimate the object area will not be covered here but can be found in $[12,13]$.

To illustrate the issue let us look at a simple example. When a randomly placed (circular) cell is digitized, one possible realization is shown in Figure 9. The equation for generating the "cell" is $\left(x-e_{x}\right)^{2}+(x-$ $\left.e_{y}\right)^{2} \leq R^{2}$ where $\mathrm{R}$ is the radius of the cell. The terms $e_{x}$ and $e_{y}$ are independent random variables with a uniform distribution over the interval $(-1 / 2,+1 / 2)$. They represent the random placement of the cell with respect to the periodic (unit) sampling grid.

In the realization shown in Fig. 9, the area would be estimated at 84 pixels. However, a slight shift of the circle with respect to the grid could change that, for example, to 81 or 83 or 86 . The sampling density of this figure can be expressed as about 10 pixels per diameter. To appreciate what effect the finite sampling density has on the area estimate, let us look at the coefficient-of-variation of the estimate, the $C V=\sigma / \mu$, where $\sigma$ is the standard deviation of the estimate of the area and $\mu$ is the average estimate over an ensemble of realizations.

If we denote the diameter of the cell by $D$, and the size of a pixel as $s \times s$, then the sampling density is $Q=D / s$. The area of the circle, $A_{l}$, that is always green (in Fig. $9)$, independent of $\left(e_{x}, e_{y}\right)$, is given by:

$$
A_{1}=\frac{\pi}{4}(D-2 s)^{2}
$$

The number of pixels associated with this is:

$$
N_{\mathrm{l}}=\frac{A_{\mathrm{t}}}{s^{2}}=\frac{\pi}{4}\left(\frac{D}{s}-2\right)^{2}=\frac{\pi}{4}(Q-2)^{2}
$$

The area of the region, $A_{b}$, that is blue (in Figure 9) is given by:

$$
A_{b}=\frac{\pi}{4}(D+2 s)^{2}-\frac{\pi}{4}(D-2 s)^{2}
$$

and the number of pixels, $N_{b}$, associated with this region is:

$$
N_{b}=\frac{\pi}{4}(Q+2)^{2}-\frac{\pi}{4}(Q-2 s)^{2}=2 \pi Q_{(9)}
$$

The area of the circle is estimated by counting pixels, and the contribution from the green region is clearly $N_{l}$. The total number will be $N_{T}=N_{l}+n$, where $n$ is a random variable. Let us make a simplifying assumption: Let us assume that each of the pixels in the blue region can be part of the object - with probability $p$, and part of the background-with probability ( 1 -

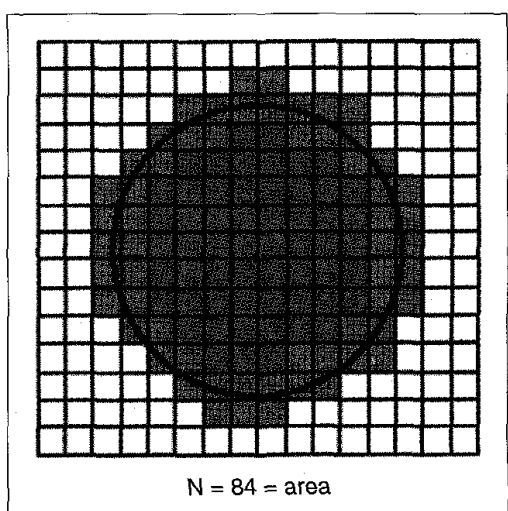

9. Given small variations in the center position of the circle, pixels that are colored green will always remain part of the object, and pixels that are colored white will always remain part of the background. Pixels that are shown in blue may change from object to background, or vice versa, depending on the specific realization of the circle center with respect to the digitizing grid. 
p) and that the decision for each pixel is independent of the other neighboring pixels in the blue region. This, of course, describes a binomial distribution for the pixels in that region. In fact, this assumption is not true and the behavior of neighboring pixels is somewhat correlated. But let us see how far we can go with this model. Under this assumption:

$$
\begin{aligned}
\mu & =E\left\{N_{T}\right\}=E\left\{N_{1}+n\right\}=N_{1}+E\{n\} \\
& =N_{1}+N_{b} p=\frac{\pi}{4}(Q-2)^{2}+2 \pi Q p
\end{aligned}
$$

and

$$
\begin{aligned}
& \sigma\left(N_{T}\right)=\sqrt{\sigma^{2}\left(N_{1}\right)+\sigma^{2}(n)}=\sigma(n) \\
& =\sqrt{N_{b} p(1-p)}=\sqrt{2 \pi Q p(1-p)}
\end{aligned}
$$

We have made use of the assumption that $N_{I}$ is deterministic-the pixels are always green-and that the mean and variance of the binomial distribution for $N_{b}$ samples with probability $p$ are given by $N_{b}$ $p$ and $N_{b} p(1-p)$, respectively.

This immediately leads to an expression for the $C V$ of our estimate as:

$$
C V=\frac{\sigma}{\mu}=\frac{\sqrt{2 \pi Q p(1-p)}}{\frac{\pi}{4}(Q-2)^{2}+2 \pi Q p}
$$

We can now study the convergence of the $\mathrm{CV}$ as the sampling density increases. As $Q$ increases in this two-dimensional image, we have:

$$
\lim _{Q \rightarrow \infty} C V(Q)=k_{2} \frac{Q^{1 / 2}}{Q^{2}}=k_{2} Q^{-3 / 2}
$$

This type of argument can easily be extended to the three-dimensional case, where the results are:

$$
C V=\frac{\sigma}{\mu}=\frac{\sqrt{\left(2 \pi Q^{2}+\frac{8 \pi}{3}\right) p(1-p)}}{\frac{\pi}{6}(Q-2)^{3}+\left(2 \pi Q^{2}+\frac{8 \pi}{3}\right) p}
$$

and

$$
\lim _{Q \rightarrow \infty} C V(Q)=k_{3} \frac{Q}{Q^{3}}=k_{3} Q^{-2}
$$
have:

Finally, for the $N$-dimensional case, we

$$
\lim _{Q \rightarrow \infty} C V(Q)=k_{N} Q^{-(N+1) / 2}
$$

The conclusion is clear. As the sampling density $Q$ increases, the precision of our estimates improve as a power of $Q$. While the independent binomial behavior cannot be strictly true, the arguments pre- sented do show the type of convergence that can be expected and how that varies with $Q$. These results have also been shown experimentally in a number of publications [11-17]. An example is shown in Fig. 10. The measurement is the volume of spheres that have been randomity placed on a sampling grid. The quality of the examining the $C V$.

It is clear from Fig. 10 that as the sampling density increases by one order of magnitude from $Q=2$ to $Q=20 \mathrm{sam}$ ples per diameter that the $C V$ decreases by two orders of magnitude. This illustrates the relation between $C V$ and $Q$ shown in equation 15 .

Choosing Sampling Density-We are now presented with an interesting conundrum. Let us say we wish to measure the area of red blood cells. Their individual diameters are on the order of $8.5 \mu \mathrm{m}$ [18]. If we use a lens with $N A=0.75$, and blue illumination with $\lambda=420 \mathrm{~nm}$ (near the absorption peak of hemoglobin), then according to Eq. 3 and the Nyquist theorem, a sampling frequency of $f_{s}>2 \cdot f_{c}=7.2$ samples per $\mu \mathrm{m}$ should be sufficient. This frequency will give around 60 samples per diameter, which according to published results [14] should lead to more than enough precision for biological work, that is, a $C V$ below the $1 \%$ level. If, however, a small chromosome, as in Fig. 4, is sampled with the same lens, then the approximate sampling density per chromosome "diameter" will be about 10 pixels, and the CV above the $1 \%$ level.

The question then becomes, should we choose the sampling density on the basis of the Nyquist sampling theorem or on the basis of the required measurement preciestimator (voxel counting) is assessed by

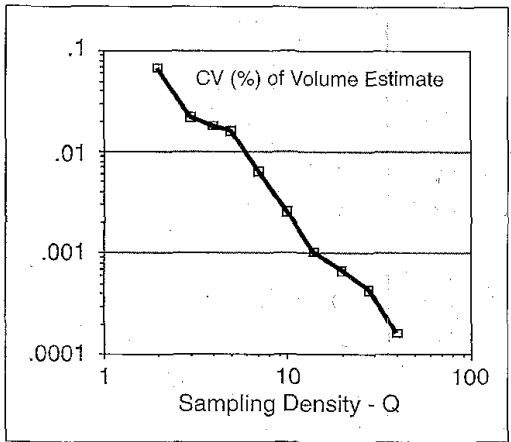

10. For each sampling density value, $Q$, expressed in voxels per diameter, 16 spheres were generated with randomly placed centers. The volume was measured by counting voxels and the $\mathrm{CV}(\mathrm{Q})=\sigma(\mathbf{Q}) / \mu(\mathbf{Q})$ calculated accordingly.

sion? The answer lies in the goal of the work. If we are interested in autofocusing or depth-of-focus or image restoration, then the Nyquist theorem should be used. If, however, we are interested in measurements derived from microscope images, then the sampling frequencies derived from measurement specifications (as exemplified in Fig. 10 and equations 13, 15, and 16) should be used.

\section{Calibration}

Finally, we come to the issue of using independent test objects and images to calibrate systems for quantitative microscopy. In this section, we will describe procedures for calculating the actual sampling density as well as the effective $C V$ for specific measurements in a quantitative microscope system.

Sampling Density-A commercially

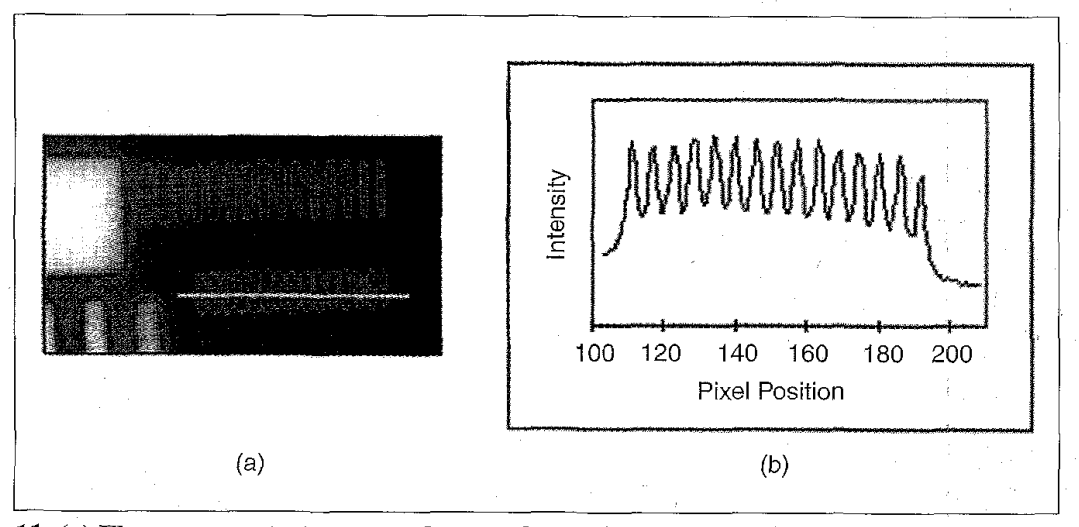

11. (a) Fluorescence test pattern that can be used to measure the sampling density. The yellow line goes through a series of bars that are known to have a $2 \mu \mathrm{m}$ center spacing $(5001 \mathrm{p} / \mathrm{mm})$. (b) The intensity profile along the yellow line indicated on the left. 


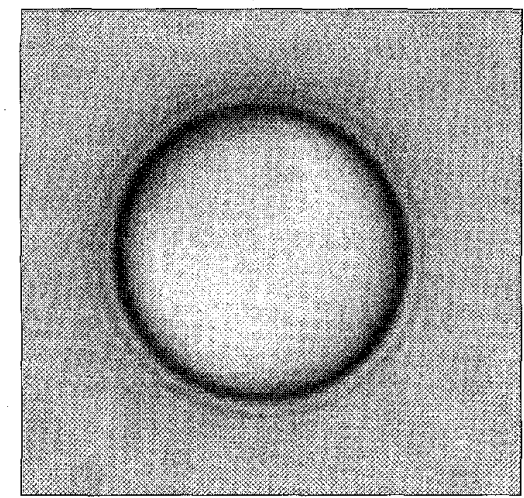

12. Fluorescently-labeled latex microsphere, observed in absorption mode with a Nikon Optiphot microscope and Nikon PlanApo 60x, NA=1.40 lens, and digitized with a Coho 4810 CCD camera (Cohu Corp., San Diego, CA), and a Data Translation QuickCapture frame grabber (Data Translation Inc., Marlboro, MA). The beads were obtained from FCSC (Flow Cyclometry Standards Corp., Research Triangle Park, NC).

prepared slide with a test pattern (a stage micrometer or a resolution test chart) is, in general, necessary to determine the sampling densities $\Delta_{\mathrm{x}}$, and $\Delta_{\mathrm{y}}$, in a microscope system and to test whether the system has square pixels, that is if $\Delta_{x}=\Delta_{y}$. An example of a digitized image of a test pattern is shown in Figure 11a. A horizontal line through a part of the image is shown in Fig. 11b. The image comes from a resolution test chart produced by Optoline (Optoline Associates, Andover, MA) and taken in fluorescence with a $63 \mathrm{x}$ lens and an $\mathrm{NA}=1.4$

Using a simple algorithm, we can process the data in Fig. 11b to determine that, averaged over 14 bars in the pattern, the sampling distance $\Delta_{\mathrm{x}}=2.9$ samples $/ \mu \mathrm{m}$. By turning the test pattern $90^{\circ}$, the sampling distance $\Delta_{\mathrm{y}}$ can be measured. Further, this test pattern can be used to compute the OTF $[3,19]$.

System performance-All measurement systems require standards for calibration, and quantitative microscopes are no exception. A useful standard is prepared samples of latex microspheres stained with various fluorescent dyes and they can also be used in absorption mode. An image of one such microsphere is shown in Fig. 12.

The sphere shown in Fig. 12 comes from a population that is characterized by the manufacturer as having an average diameter of $5.8 \mu \mathrm{m}$ and a $C V$ of $2 \%$. We can, therefore, use a population of these spheres to calibrate a quantitative system. When measuring a population for a specific property (such as diameter); we can expect a variation from sphere to sphere. The variation can be attributed to the basic instrumentation (such as electronic camera noise), the experimental procedure (such as focusing), and the "natural" variability of the microspheres. Each of these terms is independent of the others, and the total variability can therefore be written as:

$$
\sigma_{\text {total }}^{2}=\sigma_{\text {equip }}^{2}+\sigma_{\text {proc }}^{2}+\sigma_{\text {spher }}^{2}
$$

For a given average value of the desired property we have:

$$
C V_{\text {total }}^{2}=C V_{\text {equip }}^{2}+C V_{\text {proc }}^{2}+C V_{\text {spher }}^{2}
$$

Through a proper sequence of experiments, it is possible for us to assess the contribution of each of these terms to the total $C V$. This total value will then reflect the contributions from both of the effects described in detail above-the various noise sources (quantum, thermal, electronic) and the effect of the finite spatial sampling density.

As an example, let us say that we wish to examine the $C V$ associated with the measurement of the diameter of the microspheres. The diameter of these spheres can be estimated from the two-dimensional projected area of the spheres according to the estimator $\quad D=\sqrt{\frac{4 \cdot \text { Area }}{\pi}}$.

We start with a single sphere placed in the center of the microscope field-of-view and critically focused. An image is grabbed, corrected for the deterministic variation of the background illumination, and then segmented to provide a collection of labeled object pixels (we will not discuss the algorithms for background correction and proper segmentation in this article. Both topics have been discussed extensively in the literature $[20,21])$. The area and derived diameter are then determined. We then repeat this procedure, without moving the sphere, to acquire a total of $N$ estimates of the diameter. For this protocol it is clear that $\sigma_{\text {proc }}=\sigma_{\text {spher }}=$ 0 , and that only the variability associated with the equipment (the various noise sources) will contribute to the total $C V$. When this technique was applied with $N=20$ microspheres, the result was $C V_{\text {total }}$ $=C V_{\text {equip }}=0.1 \%$. Note that this value is better than one might expect on the basis of the $S N R$ per pixel, because a number of pixels were involved in determining the diameter estimate.

We now take the same microsphere and move it out of the field-of-view (in all three directions $x, y$, and $z$ ) and then back into the field at a random position. This tests the variability associated with the sampling grid as well as the effects of focusing, while keeping $\sigma_{\text {spher }}=0$. When this procedure was repeated $(N=20)$, the result was:

$$
C V_{\text {total }}=\sqrt{C V_{\text {equip }}^{2}+C V_{\text {proc }}^{2}}=0.33 \%,
$$

which means that $C V_{\text {proc }}=0.31 \%$.

We are now ready to measure the total $\mathrm{CV}$ by looking at a population of spheres. For $N=185$, the measured $C V_{\text {total }}=1.41 \%$, which means that:

$$
C V_{\text {spher }}=\sqrt{C V_{\text {total }}^{2}-\left(C V_{\text {equip }}^{2}+C V_{\text {proc }}^{2}\right)}=1.37 \%
$$

a value somewhat smaller than the manufacturer's specification. The results are summarized in Fig. 13

\section{Summary}

We have seen that modern CCD camera systems are limited by the fundamental quantum fluctuations of photons, which cannot be eliminated by "better" design. Further, proper choice of the sampling density involves not only an understanding of classic linear system theory-the Nyquist theorem-but also the equally stringent requirements of digital measurement theory. Experimental procedures that rely on the $C V$ can be used to evaluate the quality of our quantitative microscope systems and to identify which components are the "weakest link." Typical values of relatively straightforward parameters such as size can easily be measured to $\mathrm{CVs}$ around $1 \%$.

\section{Acknowledgments}

This work was partially supported by the Netherlands Organization for Scien-

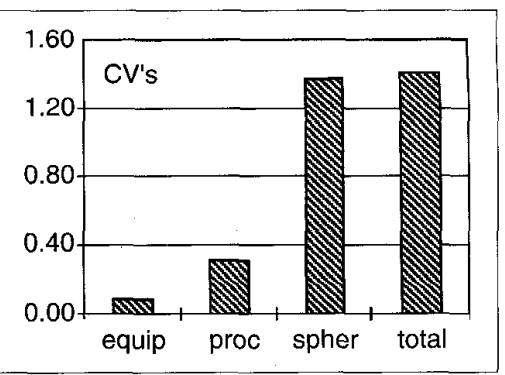

13. The various coefficients of varation, CVs, associated with the microsphere calibration protocols. 
tific Research (NWO) Grant 900-538040, the Foundation for Technical Sciences (STW) Project 2987, and the Rolling Grants program of the Foundation for Fundamental Research in Matter (FOM)

\section{References}

1. Purtle HR: History of the Microscope. Washington, DC: Armed Forces Institute of Pathology, 1974.

2. Young IT: Balasubramanian, Dunbar DL Peverini RL, and Bishop RP, "SSAM: Solid State Automated Microscope," IEEE Trans Biomed Eng, vol. BME-29: 70-82, 1982

3. Young IT: "Image Fidelity: Characterizing the Imaging Transfer Function," in Fluorescence Mi croscopy of Living Cells in Culture: Quantitative Fluorescence Microscopy -Imaging and Spectroscopy, vol. 30:B, Method in Cell Biology, D. L. Taylor and Y. L. Wang, Eds. San Diego: Academic Press, vol 30:1-45, 1989.

4. Born M and Wolf E: Principles of Optics, Sixth ed. Oxford: Pergamon Press, 1980.

5. Boddeke FR, Van Vliet LJ, Netten H, and Young IT: "Autofocusing in microscopy based on the OTF and sampling," Bioimaging, vol 2:193-203, 1995

6. Young IT, Zagers R, Van Vliet LJ, Mullikin JC, Boddeke FR, and Netten H: "Depth-of-focus in microscopy," Proceedings of the 8th Scandinavian Conference on Image Analysis, Tromso, Norway, Vol. 1:493-498, 1993.

7. Reynolds GO, DeVelis JB, Parrent BGJ, and Thompson BJ: Physical optics notebook: Tutorials in Fourier optics. Bellingham, Washington: SPIE Optical Engineering Press, 1989.

8. Marcuse D: Engineering Quantum Electrodynamics. New York: Harcourt, Brace \& World, 1970.

9. Mullikin JC, Van Vliet LJ, Netten H, Boddeke FR, Van der Feltz G, and Young IT: "Methods for CCD Camera Characterization," Proceedings of SPIE Conference on Image Acquisition and Scientific Imaging Systems, SPIE vol 2173:73-84, 1994
10. Netten H, Young IT, Prins M, Van Vliet LJ, Tanke H, Vrolijk H, and Sloos W: "Automation of Fluorescent Dot Counting in Cell Nuclei," Proceedings of 12th IAPR International Conference on Pattern Recognition, Jerusalem, Israel, IEEE Computer Society Press, pp. 84-87, 1994.

11. Matérn B: "Precision of area estimation: a numerical study," Journal of Microscopy, vol. 153:269-284, 1989.

12. Van Vliet LJ: "Grey-scale measurements in multi-dimensional digitized images," PhD Thesis, Delft University of Technology, 1993.

13. Verbeek PW and Van Vliet LJ: "Estimators of 2D edge length and position, 3D surface area and position in sampled grey-valued images," Biolmaging, vol. 1:47-61, 1993.

14. Young IT: "Sampling density and quantitative microscopy," Analytical and Quantitative Cytology and Histology, vol. 10:269-275, 1988.

15. Smeulders AWM and Dorst L: "Measurement issues in morphometry," Analytical and Quantitative Cytology and Histology, vol. 7:242$249,1985$.

16. Mullikin JC: "Discrete and continuous methods for three-dimensional image analysis," $\mathrm{PhD}$ Thesis, Delft University of Technology, 1993.

17. Mullikin JC and Verbeek PW: Surface area estimation of digitized planes, Bioimaging, vol. 1:6-16, 1993.

18. Altman PL and Dittmer DS: Blood and Other Body Fluids. Bethesda, Maryland: Federation of American Societies for Experimental Biology, 1961.

19. Young IT: "The Use of Digital Image Processing Techniques for the Calibration of Quantitative Microscopes," Proceedings of SPIE Conference on Applications of Digital Image Processing, SPIE vol 397:326-335, 1983.

20. Castleman KR: Digital Image Processing Englewood Cliffs, New Jersey: Prentice-Hall, 1979.

21. Gonzalez RC and Woods RE: Digital Image Processing. Reading, Massachusetts: AddisonWesley, 1992.
Ian T. Young was born in Chicago, Illinois in 1943. He received the B.S., M.Sc, and Ph.D. degrees, all in electrical engineering, from the Massachusetts Institute of Technology in 1965, 1966, and 1969, respectively. From 1969 to 1973 he was an Assistant Professor of Electrical Engineering and from 1973 to 1979 he was an Associate Professor of Electrical Engineering at MIT. From 1978 to 1981 he was a Group Leader for Pattern Recognition and Image Processing at the Biomedical Sciences Division of Lawrence Livermore National Laboratory, University of California, Livermore. In December 1981 he became a chaired Professor of Measurement Technology and Instrumentation Science in the Faculty of Applied Physics at the Delft University of Technology in the The Netherlands. He has also been a Visiting Professor in the Faculty of Laboratory Medicine at the University of California San Francisco and in the Electrical Engineering departments of the Technical University Delft (The Netherlands), the Technical University Linköping (Sweden), the Ecole Polytechnique Federale de Lausanne (Switzerland), the Ecole des Mines de Paris (France), and the Technical University of Bandung (Indonesia). Dr. Young is on the editorial boards of a number of scientific journals, is a coauthor of a standard textbook Signals and Systems, and is co-editor of the journal Bioimaging. Address for correspondence: Faculty of Applied Physics, Lorentzweg 1, Delft University of Technology, NL2628 CJ Delft, The Netherlands. E-mail: young@ph.tn.tudelft.nl 